\title{
New Perspective on the Augmented Taiwan Energy Flow Diagram
}

\author{
Jia-Lu Chung, Si Nguyen Tien Le, Cheng-Liang Chen \\ Department of Chemical Engineering, National Taiwan University, Taipei \\ Email: CCL@ntu.edu.tw
}

How to cite this paper: Chung, J.-L., Le, S.N.T. and Chen, C.-L. (2019) New Perspective on the Augmented Taiwan Energy Flow Diagram. Low Carbon Economy, 10, 60-81. https://doi.org/10.4236/lce.2019.102005

Received: April 9, 2019

Accepted: June 27, 2019

Published: June 30, 2019

Copyright $\odot 2019$ by author(s) and Scientific Research Publishing Inc. This work is licensed under the Creative Commons Attribution International License (CC BY 4.0).

http://creativecommons.org/licenses/by/4.0/

\section{c) (i) Open Access}

\begin{abstract}
This work promotes an advanced alternative of energy flow that is more informative and comprehensive to facilitate a better understanding of the whole national or regional energy situation. The 2017 Energy Flow of Taiwan is illustrated as an example. This innovative energy flow captures the entire picture of the utilization of primary energy for a year, therein it points out each individual primary and secondary energy supply and demand, as well as further information of the flows. Especially, it is revealed in this study the comparative efficiencies of utilization of gross energy from the difference between each providing group and corresponding receiving groups. Thus, the analysis can serve as a benchmark for the government's reference to develop or make improvements towards more efficient and better strategies of future energy use. The proposed advanced alternative of energy flow in Taiwan is suitable for promotion to analyze the energy flow in other countries or regions.
\end{abstract}

\section{Keywords}

National/Regional Energy Situation, Energy Flow, Primary Energy Supply, Electricity Production, Primary Energy/Electricity Consumption

\section{Introduction and Review of Typical Energy Flows}

The government of each country or the manager of each specific region will summarize the annual situations of that country or region in various aspects. To get good communication right in the in-depth administrative reports, it is essential to devise a clear and concise method that is easy to comprehend. Regarding to energy-related affairs as well as the national economy in a country, energy flow diagram has been employed in the annual reports of many countries' governments to express their energy situations. The situation of primary energy utilization in each country/region, of which it reflects the socioeconomic status, is a 
very distinctive feature that can be expressed in different ways. Therein, one method can be used to describe completely the whole energy situation in a dynamic expression for a country.

In the middle of decade 1970 to 1980 , the national use of product in the U.S. was firstly illustrated by Lawrence Livermore National Library (LLNL) using chart diagrams, called the Energy Flow chart [1]. The concept of this chart since then has been widespread further to apply at different extents, even at the global level, to describe the situation of energy, so-called energy flow, either carbon (or carbon dioxide potential) flow or water flow. Flow chart, named as Sankey diagram, is a single-page graphical figure where all the information and quantitative data about the resources and products, also by-products, can be found. It provides an entire picture of all related connections and complex relationships between the inputs and outputs through which viewers can find convenient and easier to analyze and comprehend the whole real situation implied in. In other words, the energy flow chart usually can be seen as a figurative diagram for a country in a particular year, including the supplies and demands connected through a network of flows, so that the energy use situation and flow situation of the country in the current year can be depicted.

Britain has drawn and issued the national energy flow charts based on their own energy statistics data for the purpose of analyzing and understanding the energy situation [2]. Especially, Japan has introduced several kinds of flow charts, such as material flow, gas engine Sankey diagram, exergy and enthalpy Sankey chart and energy Sankey diagram [3]. Energy saving is claimed to be achieved in the pulp and paper industry in Taiwan due to the analysis of energy flow where three major energy consuming mills of pulp and paper industry are analyzed to make energy saving [4]. Japanese governmental institute investigates the need from Russian supplies of oil and gas and they realize that there is a shift in energy flows to the Far East and Russian crude oil is nearing a 10\% share in the Japanese market [5]. An optimal power generation mix in Japan's nationwide power grid by considering the post-Fukushima energy policy which puts a high priority on expanding renewable energy [6]. The global flow of energy, from fuels through to the final services, is traced and focused on the technical conversion devices and passive system in each energy chain in order to identify the technical areas which are the most likely to deliver the largest efficiency gains [7].

For an individual country, the 2017 Energy Flow of the United State of American produced by Lawrence Livermore National Laboratory (LLNL) [1] is discussed in brief to illustrate the popular point of a national energy situation.

As shown in Figure 1, the supply of primary energies in the U.S. consists of conventional fossil fuels (Natural gas-Coal-Petroleum), renewable energies (Solar-Hydro-Wind-Geothermal-Biomass) and Nuclear energy, so in total of nine different primary energy terms. Electricity is kind of considered as converted energy, and thus electricity is separated from other primary energies mentioned 


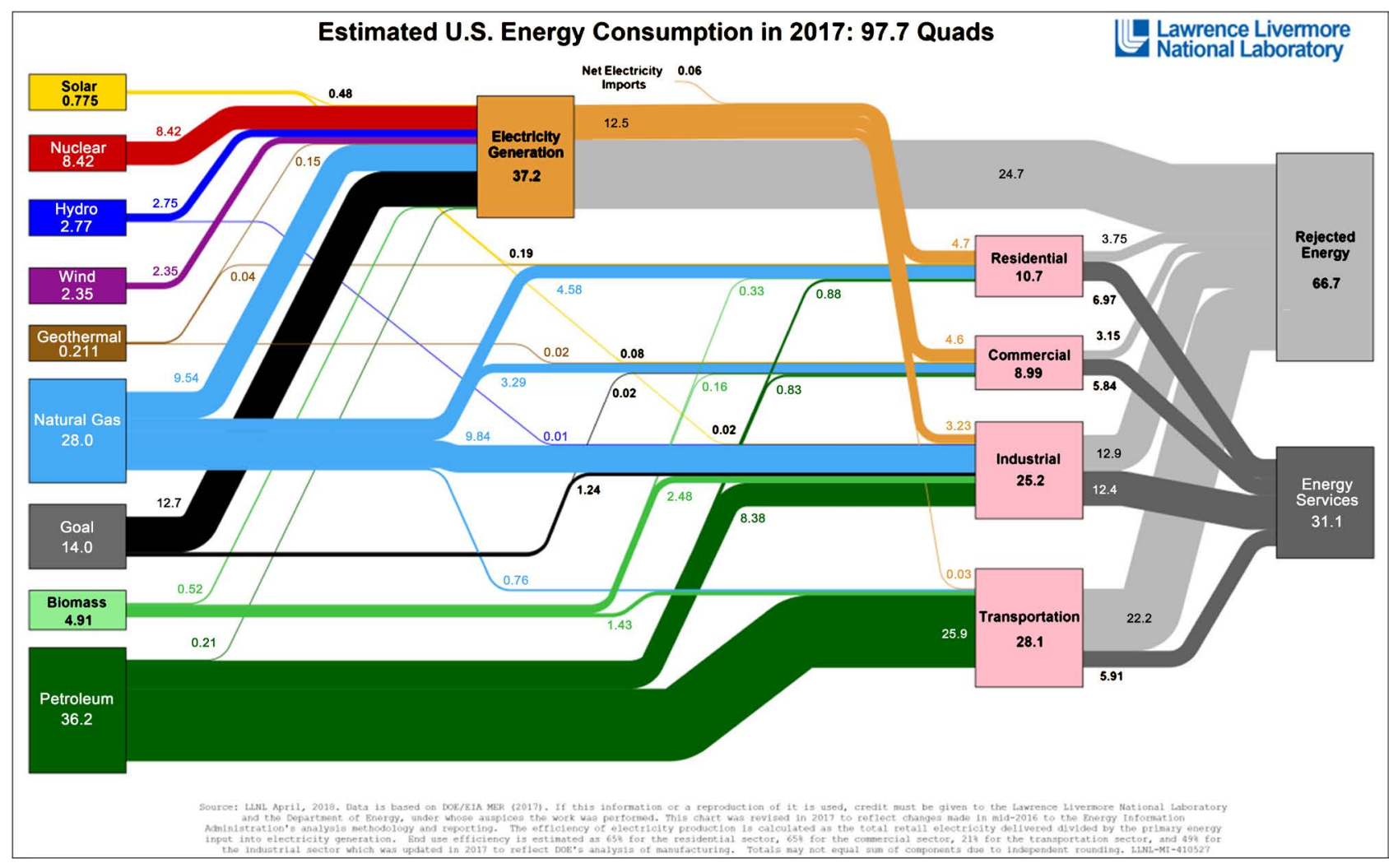

Figure 1. The 2017 Energy Flow of the United State of American [1].

above. At the same time, there are four main consumers listed including Residential, Commercial, Industry and Transportation. Moreover, based on the consumption of primary energy supply and the efficiency of the electricity consumption, as well as the data of both Rejected energy and Energy services, the utilization of primary energies can be analyzed and calculated numerically. Consequently, energy flow will reveal fine details on the situation of using various primary energies all over the whole country.

The flows of primary energies are differentiated from each other in typical width and typical color. For instance, among the total of 97.7 quads (1 quad $=1$ $\times 10^{15} \mathrm{Btu}=1.055 \times 10^{18} \mathrm{~J}=28 \mathrm{MkLOE}$, the abbreviation of Million kilo-Liter of Oil Equivalent) of primary energy supply shown in the left side, it can be obviously seen that fossil fuels such as Petroleum, Natural gas, Coal and Nuclear are those kinds of primary energies used the most. Departing from primary energy supplies along with the direction of flows, it is obvious to claim in order of priority that the dark green of Petroleum is the top supply with 36.2 quads (with a percentage of $36.2 / 97.7=37.1 \%$ ). Next is the light blue of Natural gas with 28.0 quads (28.7\%), after that the black of Coal with 14.0 quads (14.3\%) and then the red of Nuclear with 8.42 quads (8.6\%). The others show much less significant amounts. Therefore, Petroleum is the most consumed energy that the US prefers to use, $99.4 \%$ of Petroleum (35.99 quads) is shared to other uses, where Transportation is a dominant consumer, rather than the production of electricity. Obviously, Petroleum is not a favorable material for electricity pro- 
duction, there is only 0.21 quads extracted from total 36.2 quads of Petroleum, almost nothing, sent to the power generation. Meanwhile, about $90.7 \%$ (12.7 quads) of Coal is used to generate electricity, the left of Coal is divided into 1.24 quads supplied to industry and 0.02 quads supplied to commercial. Furthermore, the U.S. power generation also consumes 100\% (8.42 quads) of Nuclear and $34.1 \%$ (9.54 quads) of Natural gas. Therefore, Coal, Natural gas and Nuclear are the main materials to produce electricity in the U.S. Besides, Natural gas is actually preferred widely in other areas, such as Residential (4.58 quads), Commercial (3.29 quads), Industrial (9.84 quads), and Transportation (0.76 quads). The remained part of energy supply is renewable energy, divided as five different types of renewable energy, each of them is just a little amount that is less and much less than half of nuclear. This part is distributed evenly to all other consumption, except that Wind ( 2.35 quads) is $100 \%$ used for electricity generation.

For the electricity generation, it can be seen that the production of electricity consumes all different types of primary energies. The input of electricity production is composed of 0.48 quads of Solar, 8.42 quads of Nuclear, 2.75 quads of Hydro, 2.35 quads of Wind, 0.15 quads of Geothermal, 9.54 quads of Natural gas, 12.7 quads of Coal, 0.52 quads of Biomass, and 0.21 quads of Petroleum. Therefore, the total amount of primary energies supplied to the power generation is about 37.2 quads, equivalent to $37.2 / 97.7=38 \%$ of the total energy supply. Obviously, coal (12.7 quads) and natural gas (9.54 quads) and nuclear (8.42 quads) constitute a major portion of the input. About 12.5 quads of electricity generated is directly consumed by other demands. An estimate of the efficiency of power generation in the U.S. is computed from the data of input and output as $12.5 / 37.2=33.6 \%$. Therefore, a large part of energy, 24.7 quads, is wasted during the production of electricity. Later the discussion will mention again to this point.

The consumption of energy, including primary energies and electricity, is expressed in pink with 4 sectors: Transportation (28.1 quads) ranks first, Industrial in the close second (25.2 quads), then Residential (10.7 quads) and Commercial (8.99 quads). Therefore, the total amount of energy consumption is about 72.9 quads. For each sector of energy consumption, the contribution of energy input is described very informative. Viewers can easily find out the detailed information of every flow. Similarly, the output of every consumption sector is split into two parts: a useful part (dark color) and a useless part (less dark color). The data on each split are also attached. One minor comment, the classification of the consumption into only 4 sectors is somehow not detailed enough. Particularly, all the activities of production of the whole country included in only one sector as industrial is pretty rough and ready, hence it could not tell more details of the situation of industrial productions, but just a general image is sketched.

Additionally, it is also noticeable about the portion of Rejected energy. Rejected energy is part of energy that could be used for a purposed activity, somehow this part is not converted into the desired products but it escapes into the environment without making any benefit. It is easy to recognize that electricity 
production and Transportation contribute the largest portions to Rejected energy, 24.7 quads (37\% of total rejected energy) and 22.2 quads (33.3\%), respectively. Energy is also rejected a lot from Industrial with 12.9 quads (19.3\%). The total of useless energy is summarized about 66.7 quads. It means only $97.7-66.7=$ 31.1 quads of energy are delivered properly to all the intentions. And thus, the efficiency of the whole of the U.S energy use is about $31.8 \%(=31.1 / 97.7)$, of course, that speaks a terrible waste. Actually, the loss of energy through the power production comes from the combustion of energy material, such as Coal, to produce heat then it will be converted into mechanical energy and then into electricity. Incidentally, the transmission of electricity over long distances from large power plants to the consumers creates power losses. The major part of this loss is caused by Joule effect in transformer and power lines.

Turning to the energy consumption again, it should be brought to the attention of viewers that the sum of 4 consumption sectors is only 72.9 quads, whereas it says clearly in the title of the figure that energy consumption is 97.7 quads. Notably, the electricity produced is described in the unit of "quad". Actually, electricity should be shown in kilowatt-hour (kWh), the use of "quad" for electricity implicitly converts the electricity into an equivalence of energy. Therefore, 12.5 quads of electricity should be understood as the amount of energy successfully converted to generate electricity. And thus, the amount of energy unsuccessfully converted is considered to be rejected energy. This part of energy rejected from the electricity production is drawn directly to Rejected Energy for simplification. In fact, there is no way to separate out the energy into useful part and useless part. Hence, the output of electricity production should be sketched as only one flow. That flow will represent the real electricity with data shown in the unit of electricity, and thus the rejected energy during the generation of electricity will be included. In summary, the total energy consumption should be calculated as $72.9+24.7=97.7$ quads.

Alternatively, another perspective is proposed to figure out the issue above. Let's the value " 12.5 " of electricity describes the amount of real electricity, hence it should be 12.5 "quad ${ }_{\mathrm{e}}$ " (energy-equivalent unit of electricity: 1 quad $_{\mathrm{e}}=1.055 \times$ $10^{18} \mathrm{~J}_{\mathrm{e}}=0.293 \times 10^{12} \mathrm{kWh}=293 \mathrm{TWh}$ ), and thus it is equivalent to $3663 \mathrm{TWh}$. The use of "quad," depicts an amount of electricity implying the electricity generation efficiency $(12.5 / 37.2=33.6 \%)$. Therefore, for example, Residential sector consumes 4.7 quad $_{\mathrm{e}}$ of electricity and 6 quads of other primary energies. Because electricity and energy are not the same thing, the summation of them is meaningless ( $4.7+6=10.7$ "quad" on the Residential in Figure 1). Rather a conversion between "quad ${ }_{\mathrm{e}}$ " and "quad" in fact: using 37.2 quads of primary energies to produce 12.5 quad $_{\mathrm{e}}$ of electricity, thus $4.7 \mathrm{quad}_{\mathrm{e}}$ of electricity should have used of $4.7 \times 37.2 / 12.5=14$ quad of primary energies. Therefore, the true total consumption of Residential is $14+6=20$ quad. Similarly, considering the conversion of the unit, Industrial ranks in first place with 31.6 quad $(=21.97+3.23 \times$ $37.2 / 12.5$ or $32.3 \%$ of nationwide primary energy consumption), next is Transportation 28.2 quads $(=28.1+0.1 \times 37.2 / 12.5$ or $28.9 \%)$, Residential 20 quads 
$(=6+4.7 \times 37.2 / 12.5$ or $20.5 \%)$ and then Commercial 18.1 quad $(=4.39+4.6 \times$ $37.2 / 12.5$ or $18.5 \%)$. Consequently, the total primary energy consumption will be summed up of 97.9 quads, this result is absolutely reasonable.

In sum up, the main drawbacks of the current US Energy Flow include 1) The 4-sector classification of the consumption is somehow not detailed enough, and 2) The mix of primary energies and the energy-equivalent unit of electricity. The motivation of this work is to extend the classification of sectors of consumption, and the valid units in drawing the nationwide energy flow. The proposed new perspective on the augmented energy flow diagram will be elucidated on the preparation of Taiwan Energy Flow Diagram.

The rest of this paper is organized as follows. The current energy situation and the official energy flow in Taiwan will be reviewed and commented in Section 2. A new perspective on the augmented Taiwan Energy Flow diagram will be proposed in Section 3. And finally a concluding remark.

\section{Current Energy Situation and Energy Flow Diagram in Taiwan}

Taiwan-an Eastern Asia island-together with Singapore, Hong Kong and South Korea is known as four Asian dragons (or some may call "tigers") due to rapid industrialization, high-income economy and outstandingly strong growth rate. The island has an area of $36,000 \mathrm{~km}^{2}$ (the 136th in the world) with the population about 23 million (global 52nd). Taiwan's gross domestic product (GDP) per capita reached 22,000 USD in 2017 (international 34th), such as illustrated in Figure 2, where the data on Figure 2 and in the following discussions comes from 2017 annual report published by the Ministry of Economic Affairs in Taiwan [8].

However, this island has been deeply troubled because of natural resource

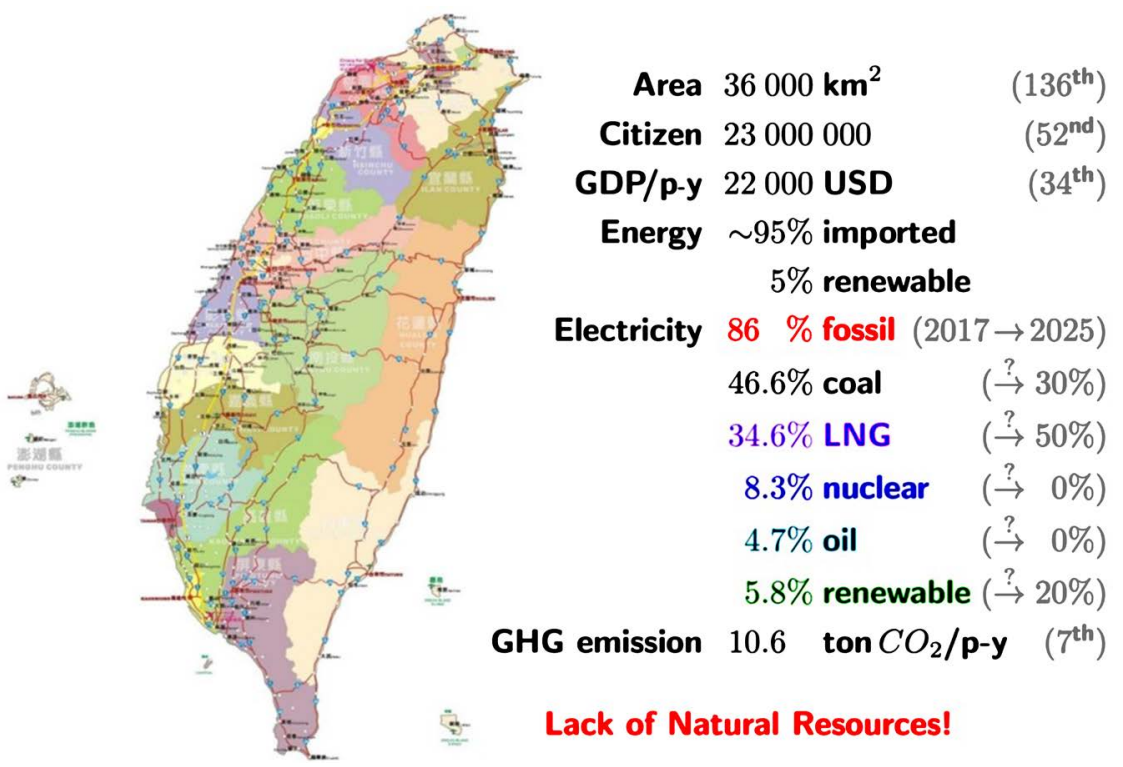

Figure 2. Current energy situation in Taiwan [8]. 
shortage. It causes a significantly high percentage of foreign energy import from other countries all over the world, $95 \%$. The rest of 5\% energy is mainly renewable energy, such as hydropower, waste, solar, and wind, etc. Furthermore, it is an essential part of the picture of energy situation in Taiwan, Taiwan's power generation (electricity production). So far, fossil fuels still play an important role in power production. There was $85.9 \%$ of energy supply, including Coal (46.6\%), LNG (34.6\%) and Oil (4.7\%) for electricity production. Besides, Nuclear power makes up $8.3 \%$ of total generated electricity, though new strategies are developed to fade out the contribution of Nuclear due to its high risk of possible uncontrolled explosion. It is claimed that until 2025, it can be eliminated the use of Oil and Nuclear for electricity production, together the use of Coal will be decreased to be about $30 \%$. The rest of $5.8 \%$ of Taiwan electricity in 2017 is generated from Renewable energy (including Conventional/Pumped hydro, Waste, and Biomass). As a result of new strategies, LNG (Liquefied Natural Gas) is expected to be a key resource accounting for $50 \%$, as well as Renewable constituting considerable proportion, $20 \%$, of energy supply for the power generation. In fact, there are two existing LNG terminal plants, one in southern Tainan and one in middle Taichung, operating at full capacities to reach the power production percentage shown above, about $34.6 \%$. In order to fulfill the goal of reaching $50 \%$ in the future, building a new LNG terminal is a deliberate attempt to increase LNG total load. As a result, Taiwan's third LNG import terminal has been approved to be constructed in the northern part of Taiwan. And the fourth one would also be possible to build up in the middle part to serve one new LNG power project. Additionally, green-house gas (GHG) emission issue is also paid serious attention to the GHG emissions reduction goals. It was reported in 2017 that there was about 10.6 tons of $\mathrm{CO}_{2}$ per Taiwanese person per year emitted to surrounding environment, ranking the 7th of the world largest emitter.

Furthermore, one can notice that if one considers gathering Coal and LNG as Group I-the main energy supplying to generate electricity and thus another Group II of Nuclear, Oil and Renewable is named. Now, let's have a quick calculation adding the percentage of each group for each year. In 2017, they are 81.2\% for Group I and 18.8\% for Group II, respectively. Meanwhile, in 2025, the total percentage is almost the same, $80 \%$ in Group I and 20\% in Group II. In other words, in the near future, Coal and LNG are still the first options to be the main source of energy for electricity production, though the priority of each type of energy is obviously changed due to the long-term energy planning. Yes, there was still 8 years to go; 8 years to reach the goal of the movement in the use of energy would not be easy if there is not enough of clear information to analyze the true situation for any solution. Therefore, there is a real need for a new way to provide more details. A rigorous thorough way to perform and describe well the whole situation is preferred to be the qualified one. Therefore, Energy Flow appeared for this reason.

The official 2017 Energy Flow of Taiwan-Figure 3-is published by MOEA 


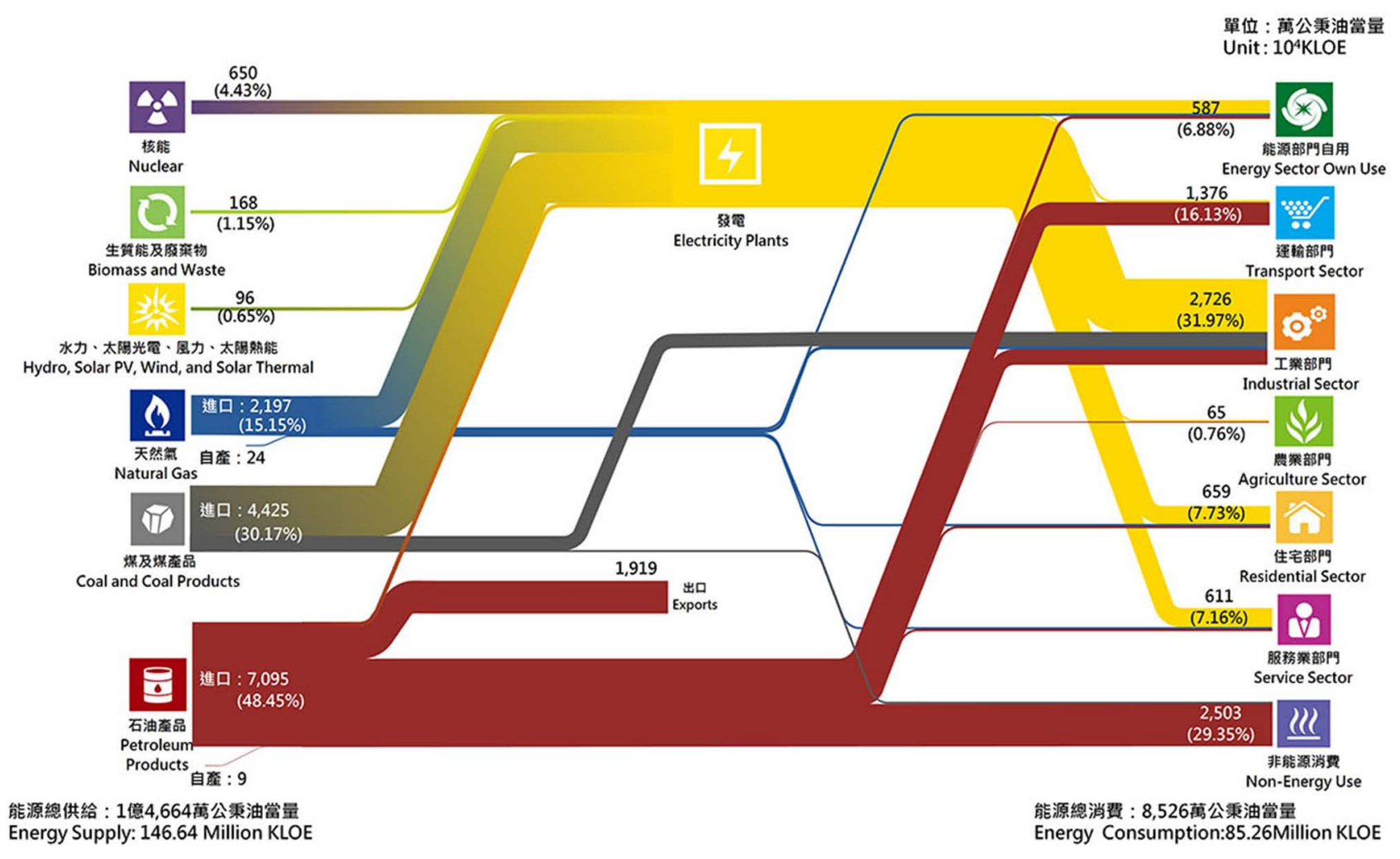

Figure 3. The official 2017 Energy Flow of Taiwan [8].

in Taiwan in the Energy Statistics Handbook 2017, though it is known with the appellation of "Energy Supply and Consumption Flowchart" [8]. As can be easily seen, it is slightly different from the U.S. Energy Flow that one element of total energy supply, namely Renewable, is preferred to list only with the subdivision instead of separating out. Besides, the energy consumption is categorized into more sectors.

Regarding to flow information, left-to-right moving direction is implicit in each flow, as well the thickness represents the portion of the flow rather than showing in numerical values. It may be very convenient to strike the use of Natural gas mainly serving the production of electricity in Taiwan. Having said that, however, it is pretty figurative and not described graphically enough to provide numerical values of flow quantity. Therefore, it is the same ambiguous to investigate further either the supply side or the demand and consumption side. In like manner, it is very obvious the total amount of consumption serves the Industrial demand the considerable largest portion, though there are such many major industrial clusters that should be singled out as a separate sector of energy consumption. In that case, it would be more conducive to grasp the full implications about the national energy situation.

The composition of the energy supply in Figure 3 is much similar to the one of U.S. that $70.95 \mathrm{MkLOE}\left(1 \mathrm{MkLOE}=37.7 \times 10^{15} \mathrm{~J}\right)$ of Petroleum (including Exports) (48.45\%), 44.25 MkLOE of Coal (43.2\%), 21.97 MkLOE of Natural Gas $(21.7 \%)$ are the main primary energy use in Taiwan. Note that LOE is ab- 
breviated for Liters of Oil Equivalent. The remains are really insignificant, therein 6.5 MkLOE of Nuclear (6.4\%), meanwhile 1.68 MkLOE of Biomass/Waste (1.6\%) and $0.96 \mathrm{MkLOE}(0.9 \%)$ of Hydro, Solar PV, Wind and Solar Thermal. Nevertheless, no more details are provided as the distribution from each source of energy supply, as well as the lack of detailed information of the splits on right side cause a big question mark implied as the electricity generation. One couldn't find any data to analyze and the production of the electricity in Taiwan through this Energy Flow. Unfortunately, there are only the values of total amount introduced as the distribution of electricity and energy to different consumption sectors. Furthermore, it is wondered whether the correction of the data is guaranteed, because there is no energy balance shown correctly. An unreasonable excess between Supply (127.36 MkLOE, being excluded from the Export of 19.19 MkLOE) and Consumption (85.27 MkLOE) is absolutely considerable. This surplus could be because of the incorrect data otherwise it is the implication of the waste of energy, $42.09 \mathrm{MkLOE}$. If that is the case, the Rejected energy can be the sum of this surplus with the Non-energy use, $42.09+25.03=67.12 \mathrm{MkLOE}$, and thus the total energy use efficiency is about $67.12 / 126.36=52.7 \%$, a quite high efficiency compared to the U.S. However, it is just a guess because there is no more information revealed to assure of the unbalance.

As can be seen, the good side of official Taiwan Energy Flow is at the point of a better classification into elements of both energy supplies and energy consumptions. Unfortunately, the shortage of detailed data, in this case, makes this official Energy Flow of Taiwan much less enlightening than the one of U.S. The first thing is already mentioned above that there is no information to describe the electricity production. Second, Non-energy Use is defined to include energy products used as raw materials in different sectors that are not consumed as a fuel nor transformed into another fuel. Thus, 25.03 MkLOE (29.35\%) of Non-energy use had better not to take into account. As a result, other consumption sectors will share equally the percentage of $70.65 \%$, equivalent to 60.24 MkLOE. Besides, it is definitely noticeable that there is such a big difference between the two consuming sectors, namely Industrial 27.26 MkLOE (31.97\%) and Agriculture $0.065 \mathrm{MkLOE}(0.76 \%)$. Although it reflects the real story that Agriculture in Taiwan only stands for a very minor percentage compared to Industrial sector from the energy consumption point of view, it would be better if there is somehow a way to subdivide Industry into smaller sectors, rearrange considering the combination with Agriculture to perform a more well-proportional look.

Regarding to the analysis of two examples above, Energy Flows of the U.S. and of Taiwan, the idea to create a more effective and informative appears to drive this work. Based on the data reported from Taiwan and the discussion in Section 1 , next section will reveal the author's work in the hope that the creation and examination on the below new Energy Flow could contribute an interesting insight into this method of energy analysis. 


\section{An Innovative Energy Flow of Taiwan in 2017}

In light of the foregoing description about Energy Flow, this work is primarily concerned about a brand new built-up of Taiwan's Energy Flow that was conceived and created by the authors. This new Energy Flow is introduced as a promising alternative to the one of the same year (in 2017) published by MOEA of Taiwan. It is expected that this new Energy Flow will be showed off as an innovative scheme that provides a better way to approach studying the circumstance of the energy utilization in Taiwan and other countries.

\subsection{Introduction of the Proposed Energy Flow}

According to data reported [8], a new figure which is called "Innovative Energy Flow" is drawn to show a whole energy situation in Taiwan with more details mentioned-Figure 4.

The description of "Innovative Energy Flow" below conducts a detailed analysis based on the numerical data from Taiwan's official report in 2017 [8]. This analysis is expected to bring out a clearer and understandable insight into a picture of energy situation in Taiwan in 2017. This analysis will be mainly highlighted with three outlines as followings:

1) Contributions of various primary energies to Electricity Production;

2) Distribution of electricity from Electricity Production to other demands;

3) Contributions of various primary energies to other demands.

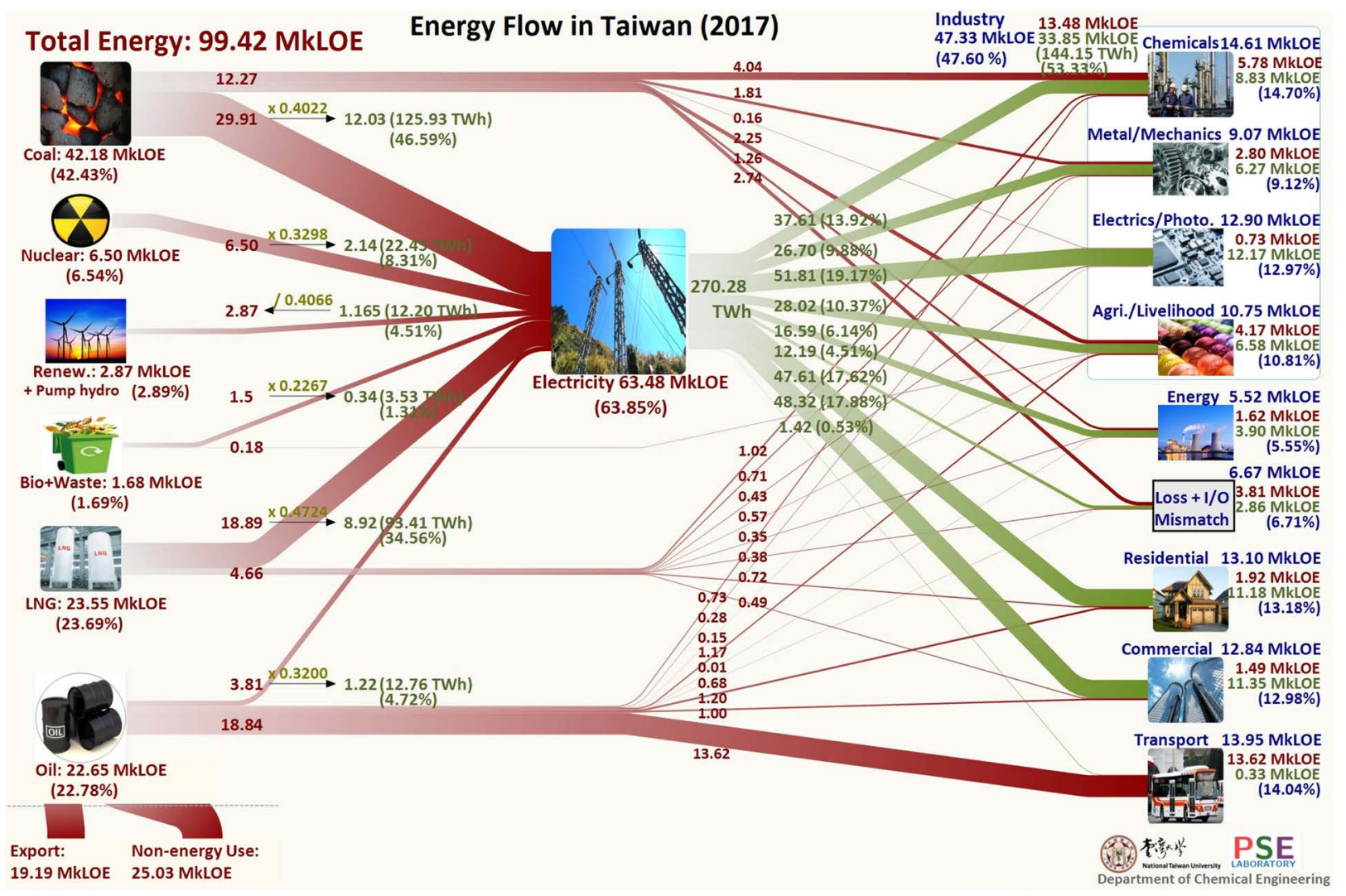

Figure 4. The proposed innovative 2017 Energy Flow of Taiwan. 
Each issue mentioned within each outline will be discussed one after another. Corresponding to three issues, there are three distinct areas over the entire figure of the innovative Energy Flow where the use of energy is described through figurative objects and numerical indications. The analysis of this Energy Flow is expected to speak well the interpretation from the figuration.

Regarding to issues listed in the outlines, it is obvious that there are three groups of subjects mentioned in general:

1) Variety of primary energies (only supply energies);

2) Electricity production (consume and supply energies);

3) Other energy consumptions (only consume energies).

First of all, it should be located these three groups on the figure of innovative Energy Flow to comprehend at first figurative translation of the outlines over the figure.

\subsubsection{Variety of Primary Energies}

The mutual characteristic among this group is only possible to supply primary energies to the demand. The first group includes all the subjects shown vertically on the left-hand side of Figure 4. It describes the variety of energy that Taiwan imported from other countries all over the world. Data attached describe the amount and the corresponding percent number of each kind of energy among the total imported energy. They are classified into six element kinds of imported primary energy, such as also shown in Table 1.

In 2017, total amount of energy supply in Taiwan is reported about 99.42 MkLOE. Remind again that the unit used in general throughout this analysis is an equivalent unit of energy "LOE"-Liters of Oil Equivalent, where 1 LOE is equivalent to $9000 \mathrm{kcal}$ or $37.6 \times 10^{6} \mathrm{~J}$ or $10.47 \mathrm{kWh}$ :

$$
\begin{aligned}
& 1 \mathrm{LOE} \approx 9000 \mathrm{kcal} \approx 37,600 \mathrm{~kJ} \approx 10.47 \mathrm{kWh} \\
& \text { and } 860 \mathrm{kcal} \approx 3600 \mathrm{~kJ} \approx 1 \mathrm{kWh}
\end{aligned}
$$

Therefore, the total amount of energy supply in Taiwan in 2017, 99.42 MkLOE, is equivalent to $3.74 \times 10^{18} \mathrm{~J}$ or $3.74 \mathrm{EJ}$. Indeed, choosing to perform the energy in the unit of MkLOE actually offers the advantage of the convenience of the similarity to the percentage since the total amount is $99.42 \mathrm{MkLOE}$,

Table 1. Supply of primary energies in 2017.

\begin{tabular}{ccc}
\hline \multirow{2}{*}{ Primary energies } & \multicolumn{2}{c}{ Total Energy Supplies } \\
\cline { 2 - 3 } Coal & MkLOE & $\%$ \\
Nuclear & 42.18 & 42.43 \\
Renewables and Pump Hydro & 6.50 & 6.54 \\
Bioenergy and Waste & 2.87 & 2.89 \\
LNG & 1.68 & 1.69 \\
Oil & 23.55 & 23.69 \\
Summation & 22.65 & 22.78 \\
\hline
\end{tabular}


an approximation of 100 percent. This also can be seen in the Energy Flow of the U.S where the unit used is "quad" to number the amount of energy as 97.7 quads, nearly 100.

\subsubsection{Electricity Production}

This typical group has a versatile characteristic that supplying energy and consuming energy are possible. This second group is subjected separately to the Electricity Production. This subject is at the middle of Figure 4 where it can be seen that a central station at which connections from the left are gathered together (all in red) and connections toward the right are spread (all in green).

It is typical over the innovative Energy Flow figure to see those lines in different colors and different thickness describing the connections between various kinds of primary energy imported from abroad and different corresponding consuming sectors. It especially distinguishes into two groups by two colors of red and green. The meaning of red is that those lines depart from any part of primary energy and they represent the contributions of various energy kinds to demanders. In other words, it can be understood as the primary energy supply within the industrial cycles in Taiwan. In the meanwhile, the meaning of green is that those lines only depart from the production of electricity and they indicate the distribution of electricity generated to other demanders. In another way, it can be considered as the internal energy supply due to production of primary energy.

\subsubsection{Other Energy Consumptions}

This group has a common characteristic of only consuming energy. The third group consists of the rest subjected to other types of industries different from Electricity industry, as well as some other typical electricity consumptions. They are all located vertically on the right-hand side of Figure 4 . There are several industries typical in Taiwan mentioned though the Energy Flow and they are grouped as a constituent of energy/electricity consumption. Below, it will show a breakdown of totally nine components in the group of only consumption, also give in Table 2. Note that the classification of the consumption into nine sectors is quite reasonable. The distribution of the data is characterized by a minor variance. In other words, the data shown in Table 2 are not at variance, it means it is fair to classify the consumption in this way. The data seems to be reasonable due to the balance of energy showing through values of total imported energy from abroad and total demanded energy domestically, 99.42 MkLOE of energy, in Taiwan 2017. Obviously, in this case, the Export and Non-energy stay outside the network of Taiwan domestic energy use.

\subsection{Contributions of Various Energy Kinds to Electricity Production}

Coming next in this section, the analysis will concentrate on the issue of electricity production. From general to detail, the analysis will discuss and provide an explanation of the data related to the utilization of imported energy including six various kinds listed above for the purpose of electricity production. 
Table 2. Total energy demands in 2017 in various sectors.

\begin{tabular}{ccc}
\hline \multirow{2}{*}{ Sectors } & \multicolumn{2}{c}{ Total Energy Demands } \\
\cline { 2 - 3 } & MkLOE & $\%$ \\
\hline Chemicals & 14.62 & 14.70 \\
Metal/ Mechanics & 9.07 & 9.12 \\
Electrics/Photoelectrics & 12.90 & 12.97 \\
Agriculture/Livelihood & 10.75 & 10.81 \\
Energy sector & 5.52 & 5.55 \\
Loss + I/O Mismatch & 6.67 & 6.71 \\
Residential & 13.10 & 13.18 \\
Commercial & 12.84 & 12.91 \\
Transport & 13.95 & 14.03 \\
Summation & 99.42 & 100 \\
\hline
\end{tabular}

Corresponding to the analysis of this section, the related area on the figure under investigation related to this issue is specified so that the analysis can be followed closely to the Energy Flow figure. Because the analysis is about to explore the electricity production in details of contributing energy, hence the attention should be kept on the center of Figure 4, the Electricity Production, as well as red lines those terminate together at the central station.

Before any further detailed discussion, for the sake of the comprehension of the analysis, it is interrupted to turn the attention to focus on some summary data of the production of electricity. After that, detailed discussion on the contributions from six kinds of primary energy to Taiwan electricity industry. A breakdown of the power production is shown in Table 3 where all the numerical data related to the production of electricity are listed.

Looking at the collected data of Taiwan electricity production, the summary was reported that the power generation required about $63.48 \mathrm{MkLOE}$ of primary energy. In other words, it can be understood that Taiwan imported 99.42 MkLOE of energy in total for domestic production and utilization, meanwhile there was part of $63.48 \mathrm{MkLOE}$ cut from the total $99.42 \mathrm{MkLOE}$ to be used for electricity production. In this way, it can be imagined that there is a pie representing the total initial primary energy. This pie is cut to divide into slices representing domestic production and utilization. And how to cut this pie properly, i.e. size of each slice cut from the pie reflects the supply of primary energy to each of corresponding demand. And hence, a proportional representative slice representing the part of total energy supply to power generation is numerically calculated as $63.48 \mathrm{MkLOE} / 99.42 \mathrm{MkLOE}$, about $63.85 \%$. So, a considerable proportion equivalent to $63.85 \%$ of the whole of the pie is cut out for the electricity slice. In percentage terms, it is obviously considerably high, the highest number among all the pie sliced slices.

Further, along with the demand of that considerable high percentage, the total 
Table 3. Primary energy supplies and used for electricity production in 2017.

\begin{tabular}{|c|c|c|c|c|c|}
\hline \multirow{2}{*}{ Primary energies } & \multirow{2}{*}{$\begin{array}{c}\text { Total } \\
\text { MkLOE }\end{array}$} & \multicolumn{2}{|c|}{ Electricity Production } & \multirow{2}{*}{$\begin{array}{c}\text { Equivalent } \\
\text { MkLOE }_{\mathrm{e}}\end{array}$} & \multirow{2}{*}{$\begin{array}{c}\text { Efficiency } \\
\%\end{array}$} \\
\hline & & MkLOE & TWh & & \\
\hline Coal & 42.18 & 29.91 & 125.93 & 12.03 & 40.22 \\
\hline$(\%)$ & $(42.43)$ & & $(46.59)$ & & \\
\hline LNG & 23.55 & 18.89 & 93.41 & 8.92 & 47.24 \\
\hline$(\%)$ & (23.69) & & $(34.56)$ & & \\
\hline Oil & 22.65 & 3.81 & 12.76 & 1.22 & 32.00 \\
\hline$(\%)$ & (22.78) & & $(4.72)$ & & \\
\hline Nuclear & 6.50 & 6.50 & 22.45 & 2.14 & 32.98 \\
\hline$(\%)$ & $(6.54)$ & & $(8.31)$ & & \\
\hline Renewables (+Pump Hydro) & 2.87 & 2.87 & 12.20 & 1.16 & 40.50 \\
\hline$(\%)$ & $(2.89)$ & & $(4.51)$ & & \\
\hline Biomaterial and Waste & 1.68 & 1.50 & 3.53 & 0.34 & 22.67 \\
\hline$(\%)$ & $(1.69)$ & & $(1.31)$ & & \\
\hline Summation or Average & 99.42 & 63.48 & 270.28 & 25.81 & 40.66 \\
\hline$(\%)$ & $(100)$ & $(63.85)$ & $(100)$ & & \\
\hline
\end{tabular}

amount of electricity production in the same year was totally of 270.28 TWh of electricity. For ease of comparing and analyzing, the total electricity-generated is converting to the unit of energy-equivalence (including efficiency), it is about 25.81 $\mathrm{MkLOE}_{\mathrm{e}}$. The following conversion will bring out the meaning of the energy-equivalent unit including efficiency:

Known that $1 \mathrm{LOE}_{\mathrm{e}} \approx 10.47 \mathrm{kWh}=10.47 \times 10^{-9} \mathrm{TWh}$

Derive that $270.28 \mathrm{TWh} \approx 2.581 \times 10^{10} \mathrm{LOE}_{\mathrm{e}}=25.81 \mathrm{MkLOE}_{\mathrm{e}}$

Implied efficiency through conversion that $\bar{E}=25.81 \mathrm{MkLOE}_{\mathrm{e}} / 63.47 \mathrm{MkLOE} \times$ $100 \%=40.66 \%$. The efficiency $\bar{E}$ calculated above is considered as the average electricity-generating efficiency and it is not expressed explicitly on the figure of Energy Flow.

Back to the point before the conversion, there was 270.28 TWh of electricity generated in total. Further review and analysis within this issue are conducted based on data related to the first group on the left-hand side. As it is introduced in the previous section, there are six kinds of energy listed as elements contributing to the industry of producing electricity in Taiwan. Standing at the first priority of the supply, as can be seen from Figure 4 and Table 3, there is 42.18 MkLOE of Coal, accounting for up to $42.41 \%$ of total energy supply. Therein, 29.91 MkLOE of Coal is used for electricity production and the rest of 12.27 MkLOE is utilized for other industries. Limited within the specified area for this issue, it is now mentioning to the amount of 29.91 MkLOE of Coal was utilized to generate electricity. Corresponding to the amount of imported Coal used to produce electricity, it is reported about 125.93 TWh of electricity generated from 
all coal-fired power plants in Taiwan. Similarly, this amount of electricity 125.93 TWh is converted into $12.03 \mathrm{MkLOE}_{\mathrm{e}}$ of energy equivalent. Thus, it is claimed that a percentage of $46.59 \%$ (125.93/270.28) of electricity is produced by Coal. Furthermore, the general efficiency for industrial processes to make electricity from Coal is calculated about $40.22 \%$ (12.03/29.91). Therefore, the first constituent divided on the electricity slice was determined as the contribution of Coal. Next, other constituents representing the contribution to electricity generation are figured out.

Other contributions are figuratively shown by some of red lines. Within the rest of red lines those represent the supply of energy to electricity generation, there are two lines noticeably catching the attention because both of them have similar thicknesses that are obviously larger than the rest. However, considering the contribution to Electricity Production, only thick red line departs from LNG seems to be potential for the second place in order of priority of contribution to Electricity industry. LNG was imported in total of 23.55 MkLOE. Correspondingly, the percentage of LNG is about $23.68 \%$ of total energy supply. However, Oil is shown with a little lower percentage of $22.78 \%$ of the total energy, equivalent to $22.65 \mathrm{MkLOE}$ - the amount of imported Oil. In general, Taiwan has used these two kinds of energy with significant imported amount every year, only less than the import of Coal. However, observing the trend of energy utilization during 20 years recently in Taiwan, LNG and Oil utilization show growing trends in the opposite manner. LNG trend in recent years is likely to increase while Oil trend is just starting downward. Obviously LNG is preferred in the circumstance of new energy strategies prior to Oil use. It can be explained that in this context of industrial production recently, LNG has shown its potential of generating the same amount of electricity but less amount of consumption compared to others, making a price-lower electricity. Moreover, the developments and improvements on LNG processing technology are offering advantages over LNG industry recently as well as in near future.

As an illustration, although sharing similar annual imports of LNG and Oil for energy use, however, considering the supply to electricity generation, supply of LNG is dramatically different from those of Oil. Especially, in 2017, while the electricity production consumed about 18.89 MkLOE of LNG, it was only 3.81 MkLOE of Oil. The difference between LNG to Oil supply to Electricity is almost five times. According to the report, LNG was used to produce of $93.41 \mathrm{TWh}$ of Electricity (34.56\% of total electricity), whilst only 12.76 TWh $(4.72 \%)$ was generated from the use of Oil. Similarly, converting these values into equivalent energy unit, they are $8.92 \mathrm{MkLOE}_{\mathrm{e}}$ and $1.22 \mathrm{MkLOE}_{\mathrm{e}}$, respectively. Therefore, the efficiency for each electricity generation process is computed of $47.24 \%$ $(=8.92 / 18.89)$ from LNG and $32.02 \%(=1.22 / 3.81)$ from Oil. It is obvious that production of electricity using LNG is more efficient and effective than using Oil. This is because part of those LNG power plants are gas turbine combined with heat recovery steam cycle. Of course, compared to the efficiency of the 
production using Coal which is $40.22 \%$, the best choice is supposed to be LNG from the generation efficiency point of view.

The input of electricity production is mentioned to include Coal (29.91 MkLOE), LNG (18.89 MkLOE) and Oil (3.81 MkLOE). Other sources of primary energy used for electricity production is listed including Nuclear (6.5 MkLOE); Renewable and Pump Hydro (Renew+Pump Hydro, 2.87 MkLOE); Biomass and Waste (Bio+Waste, $1.5 \mathrm{MkLOE}$ ). The percentage of electricity generated from these types of energy is accounted for $100 \%-46.59 \%$ (Coal) - 34.56\% (LNG) $4.72 \%(\mathrm{Oil})=14.13 \%$. Furthermore, among these three kinds of energy, the one dominated is Nuclear energy, infringing upon the influence of two others to the total situation of energy. However, in order to avoid unfortunate accidents due to the high risk of nuclear power plant, Taiwan has developed new energy strategies that tend to lessen the use of nuclear gradually till completely eliminating the need of nuclear in 2025. At the same time, it is considered to increase the proportion of LNG much higher in the near future. Considering electricity contribution, there is a little difference in the route of calculation. The supply of each kind of energy to electricity industry is still able to manage, it was reported that $6.50 \mathrm{MkLOE}$ of Nuclear and 1.5 MkLOE of Biomaterial and Waste were used for electricity generation. There was only the amount of energy supply from Renewable and Pump Hydro that was not able to determine for some reasons. Thus, Renewable will be discussed later. Regarding to Nuclear and Bio + Waste, the electricity production was measured separately for each source of energy supply. Correspondingly, there was 22.45 TWh of electricity generated from Nuclear and 3.53 TWh from Bio + Waste, which are equivalent to 2.14 $\mathrm{MkLOE}_{\mathrm{e}}$ and $0.34 \mathrm{MkLOE}_{\mathrm{e}}$ of primary energy. Therefore, the average efficiency of Nuclear power plants and Bio + Waste are 32.92\% (2.14/6.50) and 22.67\% $(0.34 / 1.5)$, respectively. The production using Nuclear or Bio + Waste to generate electricity is stated to be much less effective than the one from LNG.

In another quite different context of the power generation from Renewable and Pump Hydro, it should be noticed that the only arrow expressing in the reverse direction with all others is the right-to-left arrow describing the electricity production using Renewable and Pump Hydro. The explanation for this reverse could be that because of the characteristic of electricity production process, it is not able to measure appropriately how much electricity generated corresponding to the supply of Renewable and Pump Hydro. It means the investigation is not considered to continue with similar way as what have done above. Contrary, the calculation will start from the average electricity-generating efficiency $\bar{E}$ which is $40.66 \%$. In fact, this value is recommended based on experimental studies. Consequently, it becomes straightforward as the electricity individually generated from Renewable and Pump Hydro, which is specified of 12.20 TWh, together with the calculated average efficiency $\bar{E}$ is used to derive how much MkLOE of Renewable, as well as Pump Hydro, was required. Certainly, the unit convert is a prerequisite to convert $12.20 \mathrm{TWh}$ to equivalent $1.165 \mathrm{MkLOE}_{\mathrm{e}}$. Thereafter, it is 
computed that $1.165 \mathrm{MkLOE}_{\mathrm{e}} / 0.4066=2.87 \mathrm{MkLOE}$ of constituting primary energy supply which is categorized as Renewable and Pump Hydro energy. That is to say it is needed to use $2.87 \mathrm{MkLOE}$ of primary energy to generate the same amount of electricity of 12.20 TWh.

In short, the analysis to this point brings out the flavors of the electricity slice initially cut out from the whole primary energy pie. This slice is particularly made for the production of electricity with all the ingredients concerned through all the writing above.

\subsection{Electricity Distribution to Energy Consumptions}

In this section, the analysis will discuss a new issue of using energy as a form of electricity which is supplied from Electricity Production to other industries and to energy demands. Electricity production as mentioned above is possible either to consume energy or supply electricity. It has already analyzed the possibility of consuming energy of the central electricity production during the previous section. On the contrary, the analysis will discuss on the other side that is the possibility of supply energy which is electricity generated to meet the demands of energy. Thus, the analysis on this section only pays attention on other energy consumptions that demand for electricity, rather than any different kind of energy.

Projection of the issue in this part on the Energy Flow figure will be determined that includes the Electricity Production along with all green lines departing from the central station towards to the right till terminating at the electricity consumptions. For this Energy Flow in Taiwan, every component consuming energy demands both electricity and other energy kinds. Hence, the area determined in this analysis will include all components of energy consumption group.

Because there is no component of the third group is in the extension of this part analysis, remind that there are totally nine components in the group of only consumption. Corresponding to nine components, there are only nine green lines in Figure 4. Each green line has different thickness representing the amount of electricity generated from Electricity Production supplying to meet the demand from consumptions. Therefore, the sum of all green lines is sure to be the amount of electricity generated, 270.28 TWh, equivalent to 25.81 $\mathrm{MkLOE}_{\mathrm{e}}$ of energy.

Right next to Electricity Production on the right in the Energy Flow of Figure 4 , a set of data shown in the form of absolute values and relative percentages to describe the distribution of electricity from Electricity Production to any demand. It is quite straightforward to derive the percentage of each electricity-distributing amount. Electricity distribution data are also depicted in Table 4, where a summary of the consumption of each type of energy by each consuming sector is also included and will be discussed furthermore in next section.

The electricity distribution has met all demands from different industries and electricity consumptions. According to the data, the most use of electricity is claimed to be Electrics/Photoelectrics area, 19.17\% (51.81/270.28). Followings 
Table 4. Consumption of electricity and primary energies for each sector in 2017.

\begin{tabular}{|c|c|c|c|c|c|c|c|c|c|}
\hline \multirow{3}{*}{$\begin{array}{c}\begin{array}{c}\text { Energies } \\
\text { Sectors }\end{array} \\
\text { Chemical }\end{array}$} & Coal & Oil & LNG & Waste & \multicolumn{3}{|c|}{ Electricity } & \multicolumn{2}{|c|}{ Total energy } \\
\hline & \multicolumn{4}{|c|}{ MkLOE } & \multirow{2}{*}{$\begin{array}{l}\text { TWh } \\
37.61\end{array}$} & \multirow{2}{*}{$\begin{array}{c}\mathrm{MkLOE}_{\mathrm{e}} \\
3.59\end{array}$} & \multicolumn{2}{|c|}{ MkLOE } & \multirow{2}{*}{$\begin{array}{c}\% \\
14.70\end{array}$} \\
\hline & 4.04 & 0.73 & 1.02 & & & & 8.83 & 14.62 & \\
\hline Mechanics & 1.81 & 0.28 & 0.71 & & 26.70 & 2.55 & 6.27 & 9.07 & 9.12 \\
\hline Electrics & 0.16 & 0.15 & 0.43 & & 51.81 & 4.95 & 12.17 & 12.90 & 12.97 \\
\hline Livelihood & 2.25 & 1.17 & 0.57 & 0.18 & 28.02 & 2.68 & 6.58 & 10.75 & 10.81 \\
\hline Energy & 1.26 & 0.01 & 0.35 & & 16.59 & 1.58 & 3.90 & 5.52 & 5.55 \\
\hline IO Mismatch & 2.74 & 0.68 & 0.38 & & 12.19 & 1.16 & 2.86 & 6.67 & 6.71 \\
\hline Residential & 0 & 1.20 & 0.72 & & 47.61 & 4.55 & 11.18 & 13.10 & 13.18 \\
\hline Commercial & 0 & 1.00 & 0.49 & & 48.32 & 4.62 & 11.35 & 12.84 & 12.91 \\
\hline Transport & 0 & 13.62 & 0 & & 1.42 & 0.14 & 0.33 & 13.95 & 14.03 \\
\hline \multirow[t]{3}{*}{ Summation } & 12.26 & 18.84 & 4.67 & & 270.28 & 25.81 & 63.47 & 99.42 & 100 \\
\hline & \multicolumn{4}{|c|}{$\begin{array}{c}12.26+18.84+4.67+0.18 \\
=35.95 \mathrm{MkLOE}\end{array}$} & \multicolumn{4}{|c|}{$\begin{array}{l}\text { 63.47 MkLOE }=(270.28 \mathrm{TWh}) \\
\times\left[1 \mathrm{MkLOE}_{\mathrm{e}} / 10.47 \mathrm{TWh}\right] \\
\times\left[1 \mathrm{MkLOE} / 0.4066 \mathrm{MkLOE}_{\mathrm{e}}\right]\end{array}$} & \\
\hline & \multicolumn{8}{|c|}{ 35.95 MkLOE $+63.47 \mathrm{MkLOE}=99.42 \mathrm{MkLOE}$} & \\
\hline
\end{tabular}

are the consumptions, also at high rate, of Residential and Commercial, 17.62\% (47.61/270.28) and $17.88 \%$ (48.32/48.32), respectively. Besides, in Taiwan, major industries, such as Chemicals requires $13.92 \%$ of electricity generated (37.61/270.28); Metal and Mechanics demand 9.88\% (26.70/270.28); Agriculture and Livelihood use $10.37 \%(28.02 / 270.28)$ are sharing similar percentages of electricity produced. Consuming electricity at the lower percentage, they are listed as Energy sector 6.14\% (16.59/270.28); Loss and data Mismatch 4.51\% (12.19/270.28); Transport $0.53 \%(1.42 / 270.28)$. These data mentioned above are correspondingly revealed as green numbers locating right at the right side of Electricity Production in Figure 4, and they are all about the distribution of electricity generated from $63.85 \%$ of total primary energy used for generating electricity.

Following, the analysis will be carried further towards terminate locations of the electricity to figure out the situation of energy shown through this area of Energy Flow figure. Thus, the concentrate now is separated into each green lines heading to the right edge of Energy Flow. For each component of energy consumption on the right side of the figure, there is no doubt that the information of data is shown in the same way for each energy demand component. Therefore, it is easy to understand one component, so the rest can be figured out in a similar way. Let follow the first green line from the top of Figure 4 or the first row of Table 4, this green line represents of $37.61 \mathrm{TWh}$ (13.92\%) of electricity generated distributing to Chemicals. "Chemicals" which locates exactly at the upper right corner of the Energy Flow represents the industrial production related to chemistry, chemical and chemical engineering. "Chemicals" is considered to be consumed energy including electricity, note that in this part, only 
electricity is concerned about. According to annual report, Chemicals in Taiwan has consumed of $14.62 \mathrm{MkLOE}$ of energy, whereof electricity consumption was 37.61 TWh of electricity. Compared to the total energy (99.42 MkLOE), the demand from Chemicals accounts for about $14.70 \%$ of the total. This electricity amount of $37.61 \mathrm{TWh}$ is equivalent to $3.59 \mathrm{MkLOE}_{\mathrm{e}}(=[37.61 \mathrm{TWh} \times[1$ $\left.\left.\mathrm{MkLOE}_{\mathrm{e}} / 10.47 \mathrm{TWh}\right]\right)$ or can be converted into $8.83 \mathrm{MkLOE}(=[37.61 \mathrm{TWh}$ 1270.28 TWh] $\times$ [63.47 MkLOE]) of primary energy. The other energy supply is calculated to be $14.62-8.83=5.79 \mathrm{MkLOE}$, including 4.04 MkLOE of Coal, 1.02 MkLOE of LNG and 0.73 MkLOLE of Oil. For other consuming components, it is straightforward to find out how to obtain those all numerical information, the calculation is similar to what have done for Chemical component. Furthermore, it is just a convenient way to consider gathering the first four components from the top down together into one that including Chemicals, Metal/Mechanics, Electrics/Photoelectrics, and Agriculture/Livelihood. This new gather will represent the industrial production in Taiwan consuming electricity, or in general, demanding energy. Thus, this new block is described with data that are obtained by summing up corresponding data of four industrial components. In general, Taiwan industry consumed of $47.33 \mathrm{MkLOE}$ of total making up a percentage of $47.61 \%$ of total demand of energy. Thus, the Industry consumed 33.85 MkLOE (144.15 TWh) of Electricity and 13.48 MkLOE of primary energy.

\subsection{Contribution of Other Energy Supply for the Demand}

At this stage, the analysis brings out into the open almost two third of the Energy Flow figure. The left now is all of the rest part not mentioned yet in two previous sections. In this part, the analysis will be done for a complete understandable image about the energy situation in 2017 in Taiwan. It is the issue about the contribution of the remained amount of total primary energy towards other utilization and industrial production rather than the generation of electricity.

As can be seen on Figure 4 and Table 4, still there are red lines that run horizontally from the left to the right of the figure, though these lines seem to dim the attention because of they all are quite thin, much thinner compared to others of above discussion. By careful observation, for the direct link from the left edge to the right edge, there are four general red lines of connection departing respectively from Coal, Bio + Waste, LNG and Oil. Such as shown in Figure 4 and Table 4, they are also four other kinds of energy on demand for the consumption of all components on the right side. First, the left 12.26 MkLOE of Coal is distributed to six different demands, such as: $4.04 \mathrm{MkLOE}$ to Chemicals, $1.81 \mathrm{MkLOE}$ to Metal/Mechanics, $0.16 \mathrm{MkLOE}$ to Electrics/Photoelectrics, 2.25 MkLOE to Agriculture/Livelihood, 1.26 MkLOE to Energy sector, and 2.74 MkLOE to Loss + I/O Mismatch. Similarly, three other kinds of energy which are Bio + Waste, LNG and Oil also have a distribution over other productions rather than electricity production. While $0.18 \mathrm{MkLOE}$ of Bio + Waste is entirely supplied directly to Agriculture/Livelihood, 4.67 MkLOE of 
LNG and 18.84 MkLOE of Oil are about to be involved in more widespread use. It is proved with the evidence of a wide distribution of LNG and even a wider distribution of Oil to many different demands. The data information of LNG and Oil distribution is easy to find out on the figure. Along horizontal red lines departing from LNG and Oil towards the right till each of both lines start separating and spreading out fan-shaped, the numbers attached on each thinner line are all about the distribution for the demands.

The distribution of $4.67 \mathrm{MkLOE}$ of LNG is depicted in the following: 1.02 MkLOE to Chemicals, $0.71 \mathrm{MkLOE}$ to Metal/Mechanics, $0.43 \mathrm{MkLOE}$ to Electrics/Photoelectrics, $0.57 \mathrm{MkLOE}$ to Agriculture/Livelihood, 0.35 MkLOE to Energy, 0.38 MkLOE is Loss + Mismatch, 0.72 MkLOE to Residential, and 0.49 MkLOE to Commercial.

The distribution of $18.84 \mathrm{MkLOE}$ of Oil is: $0.73 \mathrm{MkLOE}$ to Chemicals, 0.28 MkLOE to Metal/Mechanics, 0.15 MkLOE to Electrics/Photo., 1.17 MkLOE to Agri./Livelihood, 0.01 MkLOE to Energy, 0.68 MkLOE to Loss + Mismatch, 1.20 MkLOE to Residential, $1.00 \mathrm{MkLOE}$ to Commercial, and $13.62 \mathrm{MkLOE}$ to Transport.

Terminating on the right-hand side edge of the Energy Flow figure, it is now analyzed each of energy demand component for further details. For example, Chemicals is first taken to investigate the all energy ingredients of its own demand. Beside known ingredients discussed and mentioned above, Chemicals also demands for LNG and Oil so that there are in total of 4 energy ingredients found in the demand for the recipe of chemical industrial production that in every 14.62 MkLOE of demand for Chemicals industry includes 4.04 MkLOE (27.63\%) of Coal, 1.02 MkLOE (6.98\%) of LNG, 0.73 MkLOE (4.99\%) of Oil, and 8.83 MkLOE $(60.40 \%)$ of Electricity, where the average efficiency $(40.66 \%)$ is taken into account to calculate the primary energy required for electricity production.

Next, in every 9.07 MkLOE of demand for Metal/Mechanics industry: 1.81 MkLOE (19.96\%) of Coal, 0.71 MkLOE (7.83\%) of LNG, 0.28 MkLOE (3.09\%) of Oil and 6.27 MkLOE (69.13\%) of Electricity. Similarly, in every 12.90 MkLOE of demand for Electrics/Photoelectrics: $0.16 \mathrm{MkLOE}$ (1.24\%) of Coal, 0.43 MkLOE (3.33\%) of LNG, 0.15 MkLOE (1.16\%) of Oil, and 12.17 MkLOE (94.34\%) of Electricity. In every 10.75 MkLOE of demand for Agriculture/Livelihood: 2.25 MkLOE (20.93\%) of Coal, 0.18 MkLOE (1.67\%) of Bio and Waste, 1.17 MkLOE (10.88\%) of Oil, $0.57 \mathrm{MkLOE}$ (5.30\%) of LNG, and $6.58 \mathrm{MkLOE} \mathrm{(61.21 \% )} \mathrm{of}$ Electricity. In every 5.52 MkLOE of demand for Energy related: $1.26 \mathrm{MkLOE}$ (22.83\%) of Coal, 0.35 MkLOE (6.34\%) of LNG, 0.01 MkLOE (0.18\%) of Oil, and 3.90 MkLOE (70.65\%) of Electricity. In every 6.67 MkLOE of demand for Loss and Data Mismatch: $2.74 \mathrm{MkLOE}$ (41.08\%) of Coal, $0.38 \mathrm{MkLOE} \mathrm{(5.70 \% )} \mathrm{of}$ LNG, 0.68 MkLOE (10.19\%) of Oil, and 2.86 MkLOE (42.88\%) of Electricity. In every 13.10 MkLOE of demand for Residential: 0.72 MkLOE (5.50\%) of LNG, 1.20 MkLOE (9.16\%) of Oil, and 11.18 MkLOE (85.34\%) of Electricity. In every 
12.84 MkLOE of demand for Commercial: 0.49 MkLOE (3.82\%) of LNG, 1.00 MkLOE (7.79\%) of Oil, and 11.35 MkLOE (88.39\%) of Electricity. And finally, in every 13.95 MkLOE of demand for Transport: 13.62 MkLOE (97.63\%) of Oil and $0.33 \mathrm{MkLOE}$ (2.37\%) of Electricity.

Finally, at the lower left corner of the Energy Flow diagram, Figure 4, it shows that there was 19.19 MkLOE of Oil exported in 2017 to other countries/regions, and also the amount of non-energy use of Oil was 25.03 MkLOE. Those two parts are not included in to network between energy supply and energy consumption, and thus the energy balance is guaranteed. If the energy balance is guaranteed through all mentioned above, what are Export and Non-energy? These two terms come out to notice something missed or going wrong? The answer is "No", there is nothing wrong among the analysis above, and the energy balance holds true still. That having been said that though they are shown in this innovative Taiwan Energy Flow diagram, these data are exempt from the analytical script. In fact, Oil is also utilized widely as a material for some chemical processes where the chemical products are then involved in many different manufacturing industries, like a long chain. The concept of Non-energy Use remains somewhat unclear and thus it should stay uninvolved in the field of Energy Use. Despite the vague sense of Non-energy Use, the Export is very clear that it indicates the part of energy not used domestically but exported to other countries. Formosa Petrochemical Corporation widely exports a lot of gasoline and diesel to global commercial market. For these reasons, it should be convinced that the last two terms, Export and Non-energy Use, had better to be exclusive in the analysis of the Energy Flow.

\section{Conclusion}

This work has provided an analysis, or rather a detailed description to translate the data and further information implied in the figurative Energy Flow. Through this analysis, the paper is depicted for an insightful piece to comprehend and highlight related issues about the energy situation in Taiwan in 2017 as an example. Compared to several Energy Flow figures that have been discussed in the first part, this new Energy Flow is built up from considering, learning and fixing the gaps of others. Furthermore, it also enlightens the potentials for more efficient and better strategies of energy use for each country/region in the future. It is expected that the proposed innovative approach to draw the nationwide Energy Flow diagram will be accepted and applied by more countries.

\section{Acknowledgements}

The authors thank the Ministry of Science and Technology of Taiwan for supporting this research under Grant MOST 106-3113-F-002-007.

\section{Conflicts of Interest}

The authors declare no conflicts of interest regarding the publication of this paper. 


\section{References}

[1] Lawrence Livermore National Laboratory (2011) US Energy and Carbon Flow Chart. https://flowcharts.llnl.gov/index.html

[2] Garry, P. (2008) UK Energy Flow Chart 2007. http://rs.resalliance.org/2008/09/05/uk-energy-flow-chart-2007

[3] Osaka University: Tsuji Labs (2007) Energy Sankey Diagram for Japan. http://www.sankey-diagrams.com/tag/japan

[4] Chen, H.W., Hsu, C.H. and Hong, G.B. (2012) The Case Study of Energy Flow Analysis and Strategy in Pulp and Paper Industry. Energy Policy, 43, 448-455. https://doi.org/10.1016/j.enpol.2012.01.037

[5] Motomura, M. (2014) Japan's Need for Russian Oil and Gas: A Shift in Energy Flows to the Far East. Energy Policy, 74, 68-79. https://doi.org/10.1016/j.enpol.2014.08.024

[6] Komiyama, R. and Fujii, Y. (2017) Assessment of Post-Fukushima Renewable Energy Policy in Japan’s Nation-Wide Power Grid. Energy Policy, 101, 594-611. https://doi.org/10.1016/j.enpol.2016.11.006

[7] Cullen, J.M. and Allwood, J.M. (2010) The Efficient Use of Energy: Tracing the Global Flow of Energy from Fuel to Service. Energy Policy, 38, 75-81. https://doi.org/10.1016/j.enpol.2009.08.054

[8] Bureau of Energy, Ministry of Economic Affairs Taiwan (2017) Energy Statistics Handbook. https://www.moeaboe.gov.tw/ECW_WEBPAGE/FlipBook/2017EnergySta HandBook/index.html 